\title{
X. Der Kontrollrat: Eine europäische Lösung für die Deutschlandfrage?
}

Die Geschichte des Kontrollrats in Deutschland spiegelt den Zwiespalt der Siegermächte zwischen der Destruktion Deutschlands und der Rekonstruktion Europas, zwischen nationaler Interessenpolitik und supranationaler Verflechtung, zwischen einer autonomistischen Politik der "freien Hand“ und freiwilliger Selbstbindung durch Integration wider. Der Kontrollrat galt als ein Symbol der friedenstiftenden Kooperationsfähigkeit der Vereinten Nationen über den Krieg hinaus, als Zentralpunkt einer neuen Weltfriedensordnung. Doch er erwies sich als strukturell ungeeignet für diese Aufgabe: Schon seine Organisation verführte - unabhängig von ideologischen Belastungen im beginnenden Kalten Krieg - zur ungehemmten Verfolgung nationaler Interessen. Er war nicht der Ort, an dem widerstreitende deutschlandpolitische Zielvorstellungen unter dem Druck besatzungspragmatischer Zwänge ausgeglichen wurden. Im Gegenteil: Die konkrete Entscheidungs- und Verwaltungspraxis beleuchtete tagtäglich grell die Unvereinbarkeit der west-östlichen Differenzen, die auf der Ebene der Regierungschefs oder der Außenminister von Formelkompromissen überdeckt worden war. Der Kontrollrat verwaltete Deutschland auf der Grundlage des kleinsten gemeinsamen Nenners der nationalen Großmachtinteressen; aber er gestaltete nicht: weder Deutschland noch Europa. Deutschlandpolitische Entscheidungen sind daher im Kontrollrat nicht gefallen ${ }^{1}$, sondern er exekutierte deutschlandpolitische Vorgaben der Regierungen, auf die die Militärgouverneure immer weniger Einfluß zu nehmen vermochten und die sie an dem Versuch eines kontrollratsinternen Krisenmanagements hinderten, so schmal dessen Spielräume auch gewesen sein mögen. Seine Handlungsblockade, mehr noch als sein Scheitern, schien die Rückkehr zur überholten Machtpolitik der zwanziger und dreißiger Jahre zu signalisieren. Das zu verhindern war das Ziel zahlreicher Versuche, an seine Stelle kollektive, gesamteuropäische Kontrollstrukturen zu setzen. Neuen internationalen Organisations- und Kooperationsformen wurde derart der Weg bereitet, die bei Kriegsende in den Grundzügen zwar umrissen waren, aber noch einmal zugunsten der alten Schemata verworfen wurden. In der Übergangsphase zwischen dem klassischen internationalen Mächtesystem und einem zukunftweisenden kollektiven Sicherheitssystem blieb für die deutsche Frage keine andere Lösung als die Teilung.

Die ursprüngliche Konzeption des Kontrollrats, wie sie die Briten 1944 entwickelt hatten, war eingebettet in eine ebenso kühn ausgreifende wie originäre Konzeption zur Neuordnung der globalen und europäischen Mächtebeziehungen. Im Zeichen der gemeinsamen Bedrohung durch Deutschland (und Japan), noch während des Krieges, mußten die Grundzüge der Nachkriegsordnung verbindlich festgelegt werden, solange

1 Mai, Deutschlandpolitische Entscheidungen. 
- das war Churchills Lektion aus den Erfahrungen der Zwischenkriegszeit - die Alliierten noch auf Großbritannien angewiesen waren und ehe der säkulare Verfall der britischen Weltmachtposition zu offenbar wurde: Macht mußte durch Einfluß ersetzt werden, durch präjudizierende Initiative für die gemeinsame Nachkriegsplanung in der Londoner Europäischen Beratenden Kommission ${ }^{2}$. Aus der Zusammenarbeit bei der gemeinsamen Kontrolle Deutschlands sollte sich Vertrauen zwischen den Siegern bilden, das gemeinsame Sicherheitsbedürfnis vor Deutschland der Kriegsallianz Dauerhaftigkeit verleihen, das deutsche Wirtschaftspotential als Grundlage einer koordinierten europäischen Rekonstruktion dienen. Das trug der neuen Qualität des Krieges Rechnung, die sich in der unvorstellbaren Zahl von Toten, Verwundeten und zivilen Opfern, dem organisierten Völkermord, in den riesigen Völkerverschiebungen oder in den nicht mehr bezifferbaren materiellen Schäden niederschlug. Das antizipierte gleichsam auch die neue Qualität des künftigen Friedens im Zeichen der Atombombe. Alternative, dem traditionellen Gleichgewichtsdenken entsprungene Überlegungen waren dabei nicht ausgeschlossen, notfalls durch einen „Westblock“ einen autonomen Kurs zwischen den USA und der Sowjetunion in Kooperation mit Frankreich zu steuern ${ }^{3}$. Letztere Überlegungen gewannen jedoch erst an politischem Gewicht, als die USA sich dem gesamteuropäischen Ansatz verweigerten und als sich angesichts wachsender machtpolitischer (Osteuropa) und ideologischer Differenzen die Kontrolle Deutschlands immer stärker mit der Eindämmung der Sowjetunion verknüpfte. Indem sich die ökonomische zur militärischen Eindämmung wandelte, mußte die „vorübergehende“ Teilung Deutschlands und Europas in Kauf genommen werden.

Daß alle Kriege erst nach dem Krieg gewonnen werden, hatte 1919 Arthur Moeller van den Bruck den Alliierten gedroht, besonders dem amerikanischen Präsidenten Woodrow Wilson. Dessen Nachfolger Roosevelt hatte seinerseits aus dem Scheitern der Versailler Nachkriegsordnung die Lehre gezogen, daß der Frieden bereits vor dem Friedensschluß gestaltet werden müsse; er suchte jedoch im Kriege nur die machtpolitische Ausgangsbasis, um den nachfolgenden Frieden zu gewinnen. Seine Vorstellung einer Friedensordnung war im Grunde die modernisierte Variante des europäischen Mächtekonzerts des 19. Jahrhunderts, in dem die großen Mächte durch ihr kollektives Friedensdiktat eher kriegsverhindernd als friedenstiftend wirkten. Demgegenüber suchte Cordell Hulls kapitalistische Friedensutopie über den freien Zugang zu Rohstoffen und Märkten, über unbegrenztes Wachstum und allgemeinen Wohlstand die Ursachen des Krieges zu beseitigen. Kollektive Sicherheit und konzertierter Wiederaufbau, nationale Entautarkisierung der Feindstaaten und internationale Verflechtung von Siegern und Besiegten, das war die Perspektive, wie sie vor allem die Planungsbürokratien und die diplomatischen Apparate in Washington (und ebenso in London) während des Krieges aus den Erfahrungen der zwanziger und dreißiger Jahre entwickelt hatten. Doch Roosevelts Politik der „Vertagung“ verhalf der machtpolitischen Variante zum Durchbruch; interne institutionelle Konkurrenzen zwischen State Department, Morgenthau und Militärs vereitelten die „europäische“ Lösung der Briten und ersparten der Sowjetunion den frühen Offenbarungseid ${ }^{4}$. Die vier Welt-

2 Kettenacker, Krieg, bes. Kap. V und VI.

3 Deighton, Impossible Peace. Young, Britain.

'Hammond, Directives. 
polizisten übernahmen gemeinsam die Verantwortung für die Welt als Ganzes und jeder für sich in seiner „Verantwortungssphäre“; dem korrespondierte im kleinen die Konstruktion des Alliierten Kontrollrats in Deutschland (und in Österreich). Die gemeinsame Verwaltung Deutschlands mochte als Klammer die Kooperation der Großmächte begünstigen; sollte diese sich als nicht haltbar erweisen, das war der Unterschied zu Österreich, würde eine zu starke deutsche nationale Instanz zur Fessel werden und den Prozeß der machtpolitischen Teilung beeinträchtigen oder gar verhindern. Dieses weitgefaßte Kalkül - hinter dem sich die Arroganz einer zwar interessierten, aber nicht direkt betroffenen Weltmacht verbarg, die Reparationen nicht brauchte, sondern mehr an ihrer globalen Handlungsfreiheit interessiert war - mag die Diplomaten des State Department veranlaßt haben, dem Drängen der Militärs nach Dezentralisierung nachzugeben, das sowohl auf die Verringerung der direkten Besatzungsverantwortung wie auf vorbeugende strategische Absicherung für den Fall zukünftiger Differenzen zwischen den Alliierten ausgerichtet war. Die USA unterstützten in der EAC die Sowjetunion, die an einer noch stärker dezentralisierten Lösung interessiert war, statt der britischen Forderung zu folgen, die Sowjetunion festzulegen, solange sie während des Krieges noch zum Kompromiß verurteilt schien. Obwohl die europäische Lösung in den USA selbst bei den Isolationisten wie Henry Morgenthau oder Bernard Baruch Zustimmung fand, zeichnete sich frühzeitig die immanente Sollbruchstelle ab, daß nämlich Integration als Medium der Kontrolle nicht nur einseitig den Feindstaaten als Vorleistung auferlegt werden konnte. Denn Integration, multilateral organisiert, mochte notfalls auch ohne dauerhaftes wirtschaftliches Wachstum Transparenz, Kontrolle und kollektive Sicherheit gewährleisten, begründete zugleich eine strukturelle Nichtangriffsfähigkeit, die nicht nur Anspruch an Dritte bleiben konnte, sondern auch die Großmächte binden würde. So zukunftweisend Stimsons Potsdamer Vision des späteren Marshall-Plans oder die Inter-Alliierte ReparationsAgentur als wirtschaftliche Integrationsansätze waren, so gering war zu diesem Zeitpunkt die Bereitschaft aller vier Großmächte (geworden), Beschränkungen ihrer eben erst bestätigten nationalstaatlichen Macht hinzunehmen. Nicht einmal die (Emergency) Economic Commission for Europe der Vereinten Nationen, die European Coal Organization, mehr kooperativ als integrativ angelegt, konnten die mißtrauischen Vorbehalte überwinden ${ }^{5}$. Nicht besser erging es dem Byrnes-Plan von 1945/46 zur 25jährigen Garantie der Entwaffnung Deutschlands, der noch am ehesten geeignet schien, das Provisorium des Kontrollrats in eine europäische Institution überzuleiten und zu verstetigen. Er blieb indes wegweisend und wurde über britische und französische Initiativen zum Grundstein der westeuropäischen Sicherheitsorganisationen vom Dünkirchen-Pakt bis zum NATO-Vertrag, selbst für die spätere $\mathrm{KSZE}^{6}$.

5 Wightman, Economic Co-operation.

6 Gimbel, Entmilitarisierung. Mai, American Policy, bes. S. 99. Briten und Franzosen verstanden den Plan als prinzipielle Neuorientierung der Außenpolitik der USA. PRO, FO 371/55842/C4800 (2. 5. 1946). AN, 457 (Bidault) AP 7 (14. 3. 1947). Die Idee eines Vier-Mächte-Vertrags nach dem Muster des Byrnes-Plans galt 1948 als ,dead duck“, dessen Wiederbelebung nicht im Interesse der USA war und durch regionale Sicherheits-Arrangements nach Art. 51 der UN-Charta ersetzt werden sollte. FRUS, 1948/III, S. 3 (Marshall, 10. 1. 1948), 10. NA, RG 59/ASSOA, /box 5, folder: 8031, vol. 2 (Achilles-Bérard, 13. 2. 1948). Als Weiterentwicklung des Byrnes-Plans wurden in Westeuropa Modelle einer gesamteuropäischen Sicherheitsgarantie als Rahmen für die Wiedervereinigung eines (neutralisierten) Deutschland seit 1953 neu diskutiert. AMAE, Eu(1944-60)Allemagne/950. 
Der „europäische“ Ansatz, im wesentlichen gültig bis 1947, beruhte auf drei Grundannahmen, die sich aus der außereuropäisch fundierten strategischen Autonomie der beiden angelsächsischen Weltmächte ergaben, daß nämlich am Ende eines opferreichen, erbitterten Krieges der emotionale Wunsch nach Rache hinter die rationale Suche nach tragfähigen Sicherheitsstrukturen zurücktreten müsse, daß die Sowjetunion, aber auch Frankreich, ihre durch umfangreiche Kriegszerstörungen in Frage gestellten Weltmachtansprüche bis zum Abschluß eines langwierigen Rekonstruktionsprozesses vertagen und daß beide Mächte notfalls zur Kooperation gezwungen werden müßten. Die Geschichte des Kontrollrats, wie die europäische Nachkriegsgeschichte insgesamt, hat gezeigt, daß dieser Ansatz in der gegebenen historischen Situation unrealistisch war. Der unbedingte Wille zur atomaren Parität seitens der Sowjetunion und Frankreichs ${ }^{7}$ verriet deren machtpolitischen Primat, für dessen Realisierung beide auf den ungehinderten Zugriff auf die deutschen Ressourcen bei der Wiedergewinnung ihrer strategischen Unabhängigkeit als Starthilfe nicht verzichten konnten ${ }^{8}$. Das schloß eine europäische oder kollektive Kontrolle Deutschlands durch Internationalisierung der Ruhr oder durch Umverteilung von Rohstoffen und Transportkapazitäten mit Hilfe europäischer Instanzen nicht per se aus. Ob und inwieweit aber die Sowjetunion Vorteile durch machtpolitische Pragmatik bzw. Verzicht auf ideologische Dogmatik hätte erzielen können, bleibt spekulativ, war wohl auch schon innerhalb der sowjetischen Führung sehr umstritten'. Am Ende des Krieges, als über die Organisation der Kontrolle Deutschlands und Mitteleuropas entschieden werden mußte, war die Situation viel zu offen, die zeitliche Dimension des autonomen Zugriffs im Rahmen einer ungehinderten Beutepolitik viel zu vage, als daß Frankreich und die Sowjetunion Bindungen hätten akzeptieren können, bei denen angesichts einer global nicht abgeschlossenen Machtverteilung die Nachteile einer Selbstverpflichtung kalkulierbar waren, der potentielle Nutzen jedoch ungewiß. Im Gegensatz vor allem zu den Briten erkannten die beiden kontinentalen Flügelmächte zu spät, daß die Verhinderung der gemeinsamen Verwaltung Deutschlands sie in ihrem politischen wie wirtschaftlichen Zugriff erheblich einschränkte und ihnen bestenfalls vorübergehend Vorteile bot. Die Sowjetunion hat diesen „Fehler" möglicherweise früher bemerkt als Frankreich, das an der Forderung nach Dismemberment (der Ruhr) als der politisch sichereren, aber ökonomisch zweifelhafteren Lösung festhielt. Die Sowjetunion verpaßte gleichwohl frühzeitig den Zusammenschluß mit der Bizone, weil sie letztlich die zonale politische Autonomie dem gesamtdeutschen wirtschaftlichen Gewinn vorzog, und zwar - der Eindruck ergibt sich aus den ostdeutschen und sowjetischen Akten - keineswegs nur aufgrund transformationspolitischer Engstirnigkeit, sondern eben gerade auch im Hinblick auf die „Staatsräson“ einer durch den Sieg über Deutschland selbstbewußten, aber geschwächten Weltmacht. Trotz wachsender Skepsis hat sie sich jedoch erst recht spät endgültig entschieden: im Herbst 1947. Und auch dann hat sie sich in erster Linie gegen die Einheit der Alliierten, gegen den Kontrollrat entschieden, nicht gegen die Einheit Deutschlands - in der Hoffnung auf den nationalen Behauptungswillen

7 Delmas, Naissance, S. 263 ff. Gromyko, Erinnerungen, S. 156-61.

8 Die Alternative waren ausländische, d.h. amerikanische Kredite. Das amerikanische Kreditangebot vom März 1946 war derart mit Konditionen überfrachtet, daß die Sowjetunion gar nicht darauf reagierte. Herring, Aid, S. 239.

9 Slusser, Soviet Economic Policy. 
des Bürgertums und den sozialen Befreiungswillen der „Massen“ “10. Es bedurfte eines schmerzhaften Lernprozesses sowie erheblicher amerikanischer Pressionen und Vorleistungen (Byrnes-Plan, Marshall-Plan), um für Frankreich auf europäischer Ebene akzeptabel zu machen, was es bis dahin konsequent verhindert hatte: die Wiederherstellung (kontrollierter) Staatlichkeit und (eingeschränkter) Gleichbehandlung Deutschlands, allerdings eines geteilten Deutschlands, als Voraussetzung für eine Kontrolle durch Integration. Frankreich war - ähnlich wie die UdSSR - weniger an der politischen als an der wirtschaftlichen Einheit Deutschlands interessiert. Unterhalb einer Föderation der Alliierten im Kontrollrat sollte eine Konföderation der Deutschen in weitgehend autonomen Zonen stehen, so daß durch den Dualismus von gemeinsamer Herrschaft im Kontrollrat und zonaler Handlungsfreiheit ein Maximum an Sicherheit und Kontrolle, an Reparationen und Ausbeutung gewährleistet war. Erst die potentielle Gefahr, daß durch die Teilung Deutschlands ein autonomer Weststaat entstand, der sich (und das Ruhrgebiet) französischer Einflußnahme entzog, hat Paris gezwungen, von der nationalen Nostalgie des „Poincarisme“ zur europäischen Vision Jean Monnets voranzuschreiten: von der multilateral verbrämten direkten Herrschaft über das Ruhrgebiet zur konsensualen Hegemonie durch Verflechtung.

Der „europäische“ Ansatz des Kontrollrats scheiterte insofern an den nationalen Egoismen und Interessen der Alliierten, nachdem sich die Briten mit ihrer Konzeption gegen die amerikanisch-sowjetische Koalition in der EAC nicht hatten durchsetzen können. Die entscheidenden Weichenstellungen waren bereits im September bzw. November 1944, noch weit vor der Entfremdung zwischen Ost und West, mit Zonen- und Kontrollabkommen erfolgt: nämlich Deutschland nach Zonen zu besetzen; die Entscheidung über ein Dismemberment zu vertagen, ohne sie als erledigt zu betrachten; detaillierte Festlegungen für die Zukunft und den Neuaufbau Deutschlands zu vermeiden. Dementsprechend war die Konstruktion des Kontrollrats das Ergebnis interner institutioneller Kompromisse, nicht vorausschauender Politikplanung. Gedacht als Minimalkonsens für eine kurze Übergangsphase der militärischen Besatzungsverwaltung, in der sich die realen Machtverhältnisse und Interessenslagen klären würden, blieb als kleinster gemeinsamer Nenner ein reines Organisationsstatut mit zentrifugaler Grundtendenz, indem das Schwergewicht einvernehmlich auf die zonale Autonomie der Militärgouverneure gelegt wurde. Die amerikanische Formel, daß bei fehlender Einigung im Kontrollrat jeder Zonenkommandeur das Recht zum uni-, bioder trilateralen Vorgehen habe, durchbrach das (sowjetische, letztlich nicht das französische!) Veto, präjudizierte aber zugleich die Teilung. Sie hob den Zwang zur Einigung auf - und machte dadurch den Kontrollrat lebens-, jedoch nicht handlungsfähig. Während die globalen Verantwortungssphären der Weltmächte geographisch getrennt, ökonomisch relativ autonom und staatsrechtlich nicht verknüpft waren, galt das in Deutschland nicht. Dort fehlte seit Ende Mai 1945, seit der Verhaftung der Regierung Dönitz, eine staatsrechtlich handlungsfähige deutsche Instanz, die weder durch gesamtdeutsche Parteien und Gewerkschaften ersetzt werden konnte, noch durch die auf rein wirtschaftliche Verwaltungsfunktionen reduzierten Zentralverwaltungen ersetzt werden sollte. Immerhin schien die Vernetzung der Besatzungszonen durch Infrastruktur, ökonomische Verflechtung, interregionale Handelsströme usw.

10 Nicht auszuschließen ist, daß die SED Ende 1947 die Gründung eines ostdeutschen Staates „früher, intensiver und formalisierter" als Stalin betrieben hat. Staritz, SED, S. 689. 
geeignet, auf dem Umweg über das Interesse der Siegermächte an Reparationen, Dekkung der Besatzungskosten oder Reduktion der Hilfszahlungen den Kontrollrat zu einer Hilfskonstruktion, zu einer Notbrücke, zur alliierten Klammer deutscher Einheit und Zukunft werden zu lassen.

Dieser Belastung konnte der Kontrollrat mit seinen fragilen administrativen Strukturen und seiner eingeschränkten politischen Kompetenz unmöglich gewachsen sein, zumal der Rat der Außenminister, rudimentärer Rest des kollektiven Mächtesyndikats der Weltmächte, als seine vorgesetzte Instanz sich als handlungsunfähig erwies. Gleichwohl hielten bis 1946/47 die Bemühungen an, ihn von dem übermächtig werdenden Druck durch europäische Lösungen zu entlasten. Das betraf vor allem die künftige territoriale Gestalt, die durch Zwangsumsiedlung definierte Bevölkerungszahl und die Wirtschaftsstruktur Deutschlands, die sein Industrieniveau, den Grad der möglichen Demontagen und Reparationsleistungen ${ }^{11}$ ebenso bestimmten wie seine Fähigkeit zu Ex- und Import; seine „wirtschaftliche Entmilitarisierung “12, die den Siegermächten Handelsvorteile und Sicherheit bringen mochte, durfte nicht in Chaos, Hungersnot und Bürgerkrieg umschlagen und die erstrebte Sicherheit durch einen neuen Revanchismus wieder in Frage stellen. Nachdem es sich als unmöglich erwiesen hatte, durch eine Optimierung des interzonalen Handelsaustausches auf der Ebene des Status quo eine Minimallösung durchzusetzen ${ }^{13}$, erschien unter dem Primat der europäischen Nachkriegsrekonstruktion wie unter dem Aspekt ökonomischer Rationalität eine kontrollierte Expansion des deutschen Potentials unvermeidlich, auch für die Sowjetunion, sollten deutsche Kohle, deutsche Reparationen aus laufender Produktion oder deutsche Waren in maximalem Ausmaß zur Verfügung gestellt werden. Eine solche Kontrolle - sei es durch Dekartellisierung, Sozialisierung oder Internationalisierung - mußte, wenn auf lange Sicht mit oder ohne direkte Besatzung angelegt, neue Lösungen nach sich ziehen. Über diese Ziele herrschte zweifellos Konsens zwischen den vier Alliierten; doch umstritten blieben die Mittel, die sich mit nationalen Interessen wie ideologischen Gegensätzlichkeiten verknüpften. Nicht zuletzt an der Arbeit des Kontrollrats zeigte sich, daß das Bestreben Großbritanniens und Frankreichs (aber auch der USA), die Sowjetunion von der Ruhr und damit langfristig auch aus dem Marshall-Plan fernzuhalten, ein mögliches Rapprochement der USA und der Sowjetunion verhinderte. Noch 1947 haben Teile der amerikanischen Administration eine Verknüpfung von deutscher und europäischer Ebene durch supranationale Kooperation im Kontrollrat einerseits, in der ECE und der OEEC andererseits für denkbar gehalten ${ }^{14}$. In der konzeptionellen Frühphase sollte der Marshall-Plan u. a. auch die wechselseitige Blockade Frankreichs und der Sowjetunion im Kontrollrat lösen, indem er das deutsche Problem auf eine gesamteuropäische Ebene hob und einer qualitativ anderen Lösung zuführte ${ }^{15}$. Ein Teil der Sowjetischen Militäradministration in Deutschland (SMAD) hat das, wie es scheint, zunächst in diesem Sinne akzeptiert, wie vorübergehend auch die Bildung der Bizone. Doch die Briten verweigerten seit 1946 pragmatische Einzellösungen, auch wenn sie eigenen Interessen entspra-

1 Mai, American Policy, S. 86 ff.

12 Mai, Industrielle Abrüstung.

13 Mai, Innerdeutsche Handelsbeziehungen.

14 Mai, Osthandel, S. 204 f.

15 Gimbel, Marshallplan. 
chen, mit dem Argument, deren einvernehmliche Durchführung sei ohnehin nicht gewährleistet ${ }^{16}$. Wenn es (nach der heutigen Aktenlage) einen ausdrücklichen Beschluß gab, den Kontrollrat aufzukündigen, so fiel dieser in Washington und London $^{17}$ - und zwar zugunsten einer rein westlichen, kollektiven Kontrollösung, die seit Juni 1945 als die wahrscheinlich einzig praktikable angesehen worden war, die aber erst der Marshall-Plan unverzichtbar machte.

Es wäre müßig zu rechten, ob diese Entwicklung hätte verhindert werden können. Eine an besatzungspragmatischen Zwängen orientierte Überbrückung der wachsenden Kluft in Deutschland wäre 1947 - dafür sprechen manche Anzeichen - aus dem Kontrollrat heraus noch möglich gewesen. Doch galt eine solche Entspannung zu dem Zeitpunkt in den jeweiligen Metropolen nicht mehr als die primäre Option. Dem Kontrollrat war seit der Planungsphase 1944/45 eine Organisationsform verweigert worden, die ihn nicht nur zur Klammer der Alliierten, sondern auch des Reiches hätte werden lassen können. Eine solche Funktion wäre, wenn überhaupt, nur eine deutsche Zentralregierung auszufüllen in der Lage gewesen. Doch obwohl sich die Westmächte über die Folgen weitgehend im klaren waren, haben die Briten das Ende der Regierung Dönitz zögernd in Kauf genommen, die USA aus taktischen Gründen bewußt betrieben. Deutschland besaß als einziges besetztes Land mit einem multinationalen Kontrollrat keine nationale Zentralinstanz; allein in Berlin schuf die Verknüpfung von Kommandantur und Magistrat eine bedingt arbeitsfähige Symbiose alliierter und deutscher Instanzen, die von außen, vom Kontrollrat her endgültig zerstört wurde. Trotz unterschiedlicher Bedingungen und strategischer Bedeutung demonstrierte in Österreich die Regierung Renner einen beharrlichen Gestaltungswillen der kleinen Schritte zwischen den vier Alliierten. Ebenso zeigte das Beispiel Japans, daß eine durch die Kapitulation entmachtete, aber als Regierungs- bzw. Verwaltungsinstrument genutzte Zentralinstanz rasch einen gewissen Einfluß auf die Entscheidungsfindung, mehr noch auf deren Implementierung gewinnen konnte. Durch den Potsdamer Reparationskompromiß hatten die USA selbst die Wirtschaftseinheit in Frage gestellt, sie be- bzw. verhinderten sogar die Etablierung einer deutschen QuasiRegierung („Zentralverwaltung“), obwohl damit das gesamte Konstruktionsprinzip des Kontrollrats wertlos wurde. Die Option, Deutschland zu teilen, um im Falle eines Scheiterns der Zusammenarbeit mit der Sowjetunion kooperative gesamteuropäische Strukturen durch eine regionale Machtbalance mit Deutschland und auf Kosten Deutschlands zu ersetzen, schuf ein Rückversicherungssystem, in dem der Kontrollrat keine zentrale Rolle mehr spielte. Die Teilung machte übergreifende, gesamteuropäische Lösungen für ganz Deutschland überflüssig, bannte die Gefahr, daß der Kontrollrat sich vom Hilfsorgan für eine kurze Übergangszeit zu einer anhaltenden Verpflichtung entwickelte. Damit war, schon zum Zeitpunkt seiner Einsetzung, der Kontrollrat vom Experiment zur Verlegenheitslösung verkommen. Da es - wie kaum anders zu erwarten - nicht gelang, eine neue deutsche Zentralinstanz einzurichten, waren die Alliierten gezwungen, in einem Maße Verwaltungsaufgaben zentral selbst zu übernehmen, die angesichts der widerstreitenden nationalen Interessen auf Dauer nicht in Übereinstimmung geregelt werden konnten. Die Alliierten hatten sich selbst der Möglichkeiten zu einer (punktuell durch die Besatzungspraxis gleichwohl erzwun-

16 Vgl. oben S. 242, 280 und $303 \mathrm{f}$.

17 Vgl. oben S. $302 \mathrm{ff}$. 
genen) Kooperation beraubt, allerdings - das war das Paradoxon - mit der unverbindlicheren Organisationsstruktur des Kontrollrats ein Koordinationsorgan geschaffen, das die sofortige Spaltung Deutschlands verhinderte.

Trotz aller Bedenken im Hinblick auf die Vereinbarkeit der ideologischen Prämissen und der wirtschaftlichen Interessen war die Bereitschaft der Alliierten zum pragmatischen Interessenausgleich in Deutschland zunächst ausgeprägter, als es nachträglich oft erscheinen mag. Wohl dürfte allen Beteiligten bewußt gewesen sein, daß der Kontrollrat nur bedingt in der Lage sein würde, die in Potsdam nicht gelösten Konflikte in der Praxis zu bereinigen. Aber die Alliierten - bis zu einem gewissen Grade zweifellos selbst Frankreich und die Sowjetunion - waren noch bis über die Verkündung des Marshall-Plans hinaus nach Erwartungshaltung, Interessenlogik und Machtperspektive bereit, den Zwängen eines strukturell vorgezeichneten Konflikts in Deutschland entgegenzusteuern und die Entwicklung aus unterschiedlichen nationalen Erwägungen heraus „offenzuhalten“. Der Dualismus zwischen zonaler Autonomie und zentralem Vetorecht - in der inneren Verfassung des Kontrollrats seit 1944 verankert und, anders als in Österreich ${ }^{18}$, in der Besatzungspraxis nicht aufgelöst - war von allen beteiligten Mächten dahingehend angelegt worden, nach Möglichkeit über den Kontrollrat in die anderen Zonen hineinregieren, die eigene Zone aber von externer Einflußnahme weitgehend freihalten zu können. Der fehlende Zwang zum Kompromiß forderte eine Verzögerungspolitik und die Isolation der Zonen geradezu heraus, setzte die Kontrollratsarbeit den außerdeutschen Prioritäten nationaler Interessen und Konflikte in einem Maße aus, daß an diesem sensiblen Punkt der Nachkriegsordnung der Spielraum für Kompromiß und Konzession bereits im Vorfeld stark eingeengt wurde. Der gelegentlich betonten Gradlinigkeit einer scheinbar zwangsläufigen Entwicklung bzw. einer vermeintlich zielgerichtet auf Teilung zusteuernden Politik stand der Kontrollrat als Ausdruck einer Strategie gegenüber, die die Einheit Deutschlands zumindest als ein Mittel zur Eindämmung des Gegners, als ein Loch im „Eisernen Vorhang“, als ein Instrument zur eventuellen Ausdehnung des eigenen Macht-, Einwirkungs- oder doch Einflußbereichs betrachtete. Unter diesem Aspekt sind die verschiedenen Neutralisierungspläne in den USA (Byrnes, Kennan) ${ }^{19}$, Großbritannien (Churchill 1953) ${ }^{20}$ und der Sowjetunion (Stalin-Noten 1952) ${ }^{21}$ zu bewerten, in denen - vor allem auf sowjetischer Seite - der Kontrollrat eine gewisse Rolle spielte.

Aus den besatzungspraktischen Zwängen heraus waren, ehe die Rückkehr zur Ebene europäischer Lösungsversuche eingeleitet wurde, kontrollratsimmanente Bemühungen zur Überwindung seiner Blockade entsprungen. Von der EAC und in

18 Auf Initiative de Gaulles vom Dezember 1945 gelang es, die Sowjetunion im Zweiten Kontrollabkommen vom Juni 1946 auf ein "negatives“ Veto bzw. das Veto a posteriori festzulegen, nach dem österreichische legislative Maßnahmen in Kraft traten, wenn der Kontrollrat nicht einstimmig sein Veto einlegte. Während in Deutschland der Kontrollrat Einstimmigkeit erreichen mußte, um Maßnahmen zu verabschieden, konnte in Österreich ein unilaterales Veto ein Gesamtveto des Kontrollrats verhindern. Eisterer, Frankreich und das Zweite Kontrollabkommen, in: Bischof/Leidenfrost, Bevormundete Nation, S. 187 ff. Die USA hatten zunächst „serious misgivings“ gegenüber dieser Vetoregelung und erkannten erst spät deren Bedeutung. Als die Sowjetunion versuchte, die Vetoregelung wieder rückgängig zu machen, wurde ihnen bewußt, daß das neue Abkommen „, has in practice had effort of transferring veto power from Soviet element to U.S.“ FRUS, 1946/V, S. $342 \mathrm{ff}$., 349,364

19 Frohn, Neutralisierung.

20 Foschepoth, Churchill. Steininger, Churchill.

21 Steininger, Chance. Graml, Notenkampagne. 
Potsdam politisch allein gelassen, standen angesichts des drängenden Handlungsbedarfs die Militärgouverneure ohne ein verwaltungstechnisch wie politisch aktionsfähiges Instrumentarium vor fast unlösbaren Hindernissen. Diese Notlage war am raschesten durch praktikable „Ersatzlösungen“ zu überwinden. Solange sich dabei im Herbst 1945 noch keine eindeutige Ost-West-Konstellation herausgebildet hatte, schien die Gefahr gering, daß das Provisorium zur Dauerlösung wurde:

1. Noch ehe Frankreich offiziell sein Veto gegen deutsche Zentralverwaltungen eingelegt und der Rat der Außenminister diese Frage diskutiert hatte, schlug Clay seinen Kollegen bi- bzw. trilaterale Arrangements nach der amerikanischen Interpretation des Kontrollabkommens vor, daß jeder Zonenbefehlshaber im Falle fehlender Einigung das Recht habe, ,in seiner Zone nach eigenem Gutdünken zu handeln und Abkommen mit anderen Zonenkommandeuren einzugehen, wenn er dies wünscht“. Die Kritik seines britischen Kollegen Robertson, das werde „einen Keil der Uneinigkeit in die gesamte Konzeption der Vier-Mächte-Verwaltung treiben“, hielt Clay nicht davon ab, Ende November 1945 den Briten erstmals die Gründung einer Bizone anzubieten $^{22}$. Auf der Ebene solcher bilateralen Arrangements „im Rahmen des Kontrollrats“ wurde z. B. der Interzonenhandel abgewickelt, der zum Ersatz für die fehlende Wirtschaftseinheit wurde.

2. Bereits im Potsdamer Abkommen wurde das Prinzip verankert, durch die Trennung der wirtschaftlichen von der politischen Einheit wenigstens die nationalen Reparations- und Ausbeutungsinteressen wahrnehmen zu können, ohne sich in der Frage des Dismemberment, der deutschen Grenzen und der Formen der inneren Organisation Deutschlands festlegen zu müssen. Die deutschen Zentralverwaltungen waren allein auf die Wirtschaftseinheit zugeschnitten, doch wurde diese zeitgleich in Potsdam durch den Reparationskompromiß aufgesprengt, nach dem sich die Sowjetunion im wesentlichen aus ihrer eigenen Zone bedienen sollte. Angesichts fehlender deutscher Zentralinstanzen sah sich der Kontrollrat gezwungen, selbst Regulierungs- und Verwaltungstätigkeiten zu übernehmen, um wenigstens in Ansätzen dem Postulat der Wirtschaftseinheit nachzukommen. Rohstoffe (Eisen, Stahl, Kohle) und Energie, anfangs auch Saatgut oder Zuchtvieh, wurden relativ gleichmäßig auf die Zonen verteilt, aber nicht in einer interzonalen „Handelsbilanz“ gegeneinander aufgerechnet. Nachdem die Sowjetunion noch vor Potsdam einseitig die agrarischen Ostgebiete und die schlesische Kohle dem Zuständigkeitsbereich des Kontrollrats entzogen hatte, lag indes der geographische Vorteil auf seiten der Westmächte. Während die IG Farben so „ungünstig“ über die vier Zonen verteilt war, daß die wechselseitigen Abhängigkeiten der Betriebsteile des unter Vier-Mächte-Sequester stehenden Konzerns bis 1948 nicht vollständig gelöst worden waren (und selbst in der Frühphase der Berlin-Blockade nicht gelöst wurden), konzentrierten sich Steinkohlevorkommen und Stahlproduktion weitgehend in den Westzonen, besonders im Ruhrgebiet. Als die Westmächte den Vorteil nutzten, um angesichts ihrer Abhängigkeit von diesen Wirtschaftsgütern (im Hinblick auf ihr Reparationsprogramm) die Sowjetunion zu einer gewissen „Öffnung“ der SBZ zu zwingen, zog diese es vor, den ökonomischen Vorteil der politischen Autonomie zu opfern. In der währungs- und interzonenhandelspolitischen Abkoppelung lag die eigentliche Brisanz der Bizone, die von der Sowjetunion erst mit einer gewis-

22 Vgl. oben S. $94 \mathrm{f}$. 
sen Verzögerung attackiert wurde. Mit der Bizone, d. h. dem Verzicht auf die Zentralverwaltungen, hatten sich die Briten und Amerikaner dem französischen und sowjetischen Ansatz angeschlossen: nämlich durch informelle, interzonale Kooperation ein Maximum an Vorteilen zu ziehen, ohne sich auf politische Vor-bzw. Gegenleistungen festlegen zu müssen.

3. Insofern waren alle Überlegungen wenig aussichtsreich, die Blockade des Kontrollrats, seine Überlastung mit direkten Verwaltungsaufgaben, seine Überlagerung durch nationale Interessen der Alliierten durch eine Übertragung von Verantwortlichkeiten auf die Deutschen zu überwinden. Das war zunehmend Praxis in allen Zonen, blieb aber auch Hintergrund für die anhaltenden Bemühungen, doch noch in der einen oder anderen Form deutsche Zentralverwaltungen zu errichten. Die französische Alternative der Bureaux alliés, als von den Alliierten selbst (wenngleich mit deutscher Verwaltungsunterstützung) betriebene Übergangslösung, war in Ansätzen bereits vor Potsdam erkennbar und wurde selbst von der großen Mehrheit der französischen Kontrollratsgruppe als ungenügend verworfen. Während de Gaulle und seine Gefolgsleute, Außenminister Bidault sowie der Oberkommandierende Koenig, fast im Alleingang die Zentralverwaltungen verhinderten, blockierte auf der anderen Seite Clay in einer einsamen Entscheidung die Bureaux alliés, obwohl Spitzenbeamte des OMGUS seit dem Herbst 1945 an der Entwicklung dieser Ersatzlösung beteiligt gewesen waren. Ob allerdings selbst bei einem innerwestlichen Konsens die Zentralverwaltungen oder die Bureaux alliés von der Sowjetunion akzeptiert worden wären, erscheint im Lichte ihrer Strategie im Kontrollrat eher unwahrscheinlich, sofern diese das Recht haben sollten, in die SBZ hineinzuregieren, und derart das Vetorecht des Zonenkommandeurs ausgehöhlt hätten. Der Versuch, die Blockade des Kontrollrats durch eine deutsche „Ersatzlösung“ aufzubrechen, war mehr eine Geste der Hilflosigkeit und der Propaganda. Die Münchner Ministerpräsidentenkonferenz vom Juni 1947 scheiterte an der taktischen wie ideologischen Überlastung der Verhandlungen ebenso wie an dem Unwillen und/oder der Unfähigkeit der Deutschen, mehr als nur „Ministerpräsidenten der Alliierten " zu sein. Die Wiedervereinigungspolitik der Sowjetunion in den fünfziger Jahren, inkl. der Stalin-Noten, war ein einziger Versuch, den Fehler nachträglich zu korrigieren, daß sie seit 1947 auf den nationalen Einheitswillen der Deutschen bzw. des deutschen Bürgertums gesetzt hatte. Doch beruhten die Angebote auf einem zwangsläufig folgenden zweiten Fehlschluß: Die sowjetische Führung übersah, daß infolge ihres ersten Fehlers der Westen politisch und ökonomisch sich derart konsolidiert hatte, daß er weder Ostdeutschland noch Osteuropa zum Überleben brauchte. Bis zum 17. Juni 1953 hat sich die Sowjetunion Illusionen über die eigene deutschlandpolitische Handlungsfähigkeit gemacht, gleichzeitig den Kompromißzwang für den Westen infolge ideologischer Prämissen in grotesker Form überschätzt: Die Spekulation auf die „Krisenhaftigkeit“ der westlichen Entwicklung war ebenso verfehlt wie der Glaube an den "nationalen“ Willen der Deutschen. Erst spät, zu spät, hat die UdSSR den potentiellen Gewinn einer europäischen Lösung der Deutschlandfrage erkannt: mit dem Angebot der Neutralisierung, verknüpft mit einem handels- ${ }^{23}$ und einem sicherheitspolitischen (KSZE) Gesamtrahmen ${ }^{24}$.

23 Mai, Osthandel, S. 205.

24 FRUS, 1952-54/VII, S. 1182-92. 
4. Aber auch eine alliierte Lösung auf Kosten der Deutschen erwies sich als aussichtslos: Der Versuch Clays und seines sowjetischen Kollegen Sokolowski, d. h. der Vertreter der beiden Vormächte, die Wirtschaftseinheit gegen die Zusage von Reparationen aus laufender Produktion einzutauschen, war durch Sondierungen Sokolowskis im August 1946 eingeleitet worden, allerdings belastet durch einen gegenläufigen Kurswechsel der Moskauer Zentrale in der Reparationspolitik. Wie die französische, so war auch die sowjetische Politik gezwungen, nach der radikalen Ausbeutung ihrer Zone den Zugriff auf die gesamtdeutschen Ressourcen zu suchen, ohne die ausschließliche Kontrolle des eigenen Einflußbereichs preiszugeben. Der Kontrollrat als Medium alliierter Einheit und deutscher Teilung war für einen solchen Ansatz unverzichtbar. Doch kam diese Einsicht in beiden Fällen im Sommer 1946 zu spät. Politisch gescheitert sind diese Versuche fast zwangsläufig auf der Moskauer Außenministerkonferenz 1947, und zwar am Widerstand der jeweiligen Regierungen, obwohl Clay und Sokolowski, entmutigt und daher ein wenig halbherzig, die Alternative weiterverfolgten. Nur auf dieser Ebene wäre 1947 noch ein Durchbruch im Kontrollrat und $d u r c b$ den Kontrollrat denkbar gewesen. Aber so, wie für die Sowjetunion die Reparationsfrage der Kernpunkt war, so zentral war für die Westmächte die Währungsreform. Beide Bereiche waren, nach dem Marshall-Plan zumal, Ende 1947 nicht mehr auf einen Nenner zu bringen: Es mußte eine Entscheidung gefällt werden zwischen dem Primat der Rekonstruktion der (kriegszerstörten) alliierten Staaten und dem der Rekonstruktion Deutschlands.

An diesem Punkt wurde die prinzipielle Differenz zwischen Konsensbereitschaft und Konsensfähigkeit deutlich - bei allen vier Mächten, besonders aber bei der Sowjetunion. Zwar gingen bereits am 5. Juni 1945, als der erste Anlauf der Westmächte zur Gründung des Kontrollrats scheiterte, alle vier Mächte von der Erwartung bzw. Befürchtung aus, es werde zur Teilung Deutschlands zwischen Ost und West kommen. Man darf diese ersten, aus der aktuellen Enttäuschung entsprungenen Äußerungen nicht fehlinterpretieren als Entschluß zur Teilung. Aber der skeptische Vorbehalt begründete abwartende Zurückhaltung, schränkte die Kompromißfähigkeit ein, erforderte geradezu den Ausbau der eigenen Machtpositionen in Deutschland als Voraussetzung jeder ernsthaften Verhandlung: Besonders die Sowjetunion hat durch die in ihrer Zone betriebene Politik der Präjudizierung alles getan, die befürchteten Spaltungstendenzen zu beschleunigen. Die nach übereilt getroffenem Beschluß vom 4. Juni 1945 nur eine Woche später erfolgte Zulassung von Parteien und Gewerkschaften in der SBZ hat konkurrierende Neugründungen in den Westzonen provoziert, bei den westlichen Alliierten das Mißtrauen vertieft, das Bestätigung fand, je mehr Parteien und Gewerkschaften im Osten zu Instrumenten der Transformation wurden. Je länger das zögernde Beharren auf unilateraler alliierter Selbst-Verwaltung in den vier Zonen dauerte, je stärker dieser Vorbehalt um die Sympathien der Deutschen warb, je deutlicher die Verzögerungen besatzungspraktische Zwänge auslösten, um so größer wurde der Druck, nach dem eigenen Gesellschaftsmodell in die Reorganisation bzw. Transformation einzutreten. Da der Spirale von unilateraler Handlungsautonomie in den Zonen und nachträglicher Angleichungs- bzw. Korrekturversuche des Kontrollrats kein deutsches Korrektiv gegenüberstand, erwies sich die Befürchtung einer möglichen Teilung Deutschlands letztlich als „self-fulfilling prophecy“: Dem aus der Nostalgie der Kriegskoalition entspringenden Willen bzw. dem besat- 
zungspraktischen Zwang zur Kooperation standen taktische Politikunfähigkeit und ideologische Vertrauenssperre gegenüber. Mit Zonen- und Kontrollabkommen waren die deutschlandpolitisch präjudizierenden Vorentscheidungen längst gefallen. Der politische Entschluß, diesen Schritt auch zu vollziehen, erfolgte fast zwangsläufig: lange vertagt, war die Teilung doch angesichts der nationalen Interessen, der ideologischen Differenzen und der besatzungspraktischen Sachlogik unvermeidlich.

Die bemerkenswertesten Beispiele wechselseitiger Kooperationswilligkeit, aber faktischer Kooperationsunfähigkeit waren die Reparationen aus laufender Produktion und die Zentralverwaltungen. Obwohl die Sowjetunion ihre Angebote, die Blockade des Kontrollrats zu überwinden, fast ausschließlich im Hinblick auf ihre Reparationsinteressen machte, als Gegenleistung die Notwendigkeit von Zugeständnissen etwa im Bereich der Wirtschaftseinheit akzeptierte, war sie nicht in der Lage, den entscheidenden Schritt zu tun: nämlich den unmittelbaren Zugriff auf ihre wichtigste Quelle für Reparationen aus laufender Produktion, die Sowjet AGs, aufzugeben, um dafür den (direkten oder indirekten) Zugriff auf das symbolisch, strategisch und ökonomisch viel interessantere Ruhrgebiet einzutauschen. Kaum anders Briten und Franzosen, die sowohl selbst an Lieferungen aus der laufenden Produktion interessiert waren als auch durch die Herstellung der Wirtschaftseinheit ihre Besatzungskosten hätten reduzieren können. Für die USA lagen zumindest die politischen Vorteile auf der Hand: die Durchbrechung des „Eisernen Vorhangs“, die Lockerung des direkten Zugriffs der Sowjetunion auf ihre Zone, ein kleines Stück „roll-back“ - wie in Österreich. Mißtrauen, Prestigedenken, politischer Poker auf Kosten der Deutschen verhinderten indes Vorleistungen. Alle Seiten haben im Grunde die Vorteile eines Kompromisses erkannt; aber allein die Tatsache, daß auch die Gegenseite in der Lage war, diesem zuzustimmen, hat dessen Ablehnung bewirkt: weil das als Zeichen der Schwäche gedeutet wurde, die es durch Nachforderungen auszukosten galt. Die Westmächte haben seit Potsdam versucht, die totale Konzentration der Sowjetunion auf die Reparationsfrage als Handelsobjekt auszunutzen, ohne zu erkennen, daß diese, die sich als die eigentliche Siegermacht gegenüber Deutschland empfand, angesichts ihres Anspruchs auf weltpolitische Parität derartige Bedingungen nicht akzeptieren konnte, selbst unabhängig von dem ideologischen Gegensatz. Intentional hat die Sowjetunion lange an ihre Kooperationsfähigkeit geglaubt, obwohl ihre Besatzungspolitik längst nicht mehr konsens- und kooperationsfähig war. Der immanente Konflikt zwischen Ideologen (Intentionalisten) und Besatzungspragmatikern (Funktionalisten) machte die sowjetische Deutschland- und Außenpolitik unberechenbar, ambivalent - und illusionär.

Wie nachdrücklich die Distanz der Westmächte zur Sowjetunion von kultureller Fremdheit, machtpolitischer Konkurrenz und ideologischer Grunddifferenz bestimmt wurde, zeigte das innerwestliche Verhältnis, vor allem zwischen den USA und Frankreich. Die französische Politik war nicht minder ambivalent ${ }^{25}$ und widersprüchlich, da bei dem maßgeblichen Trio von Präsident de Gaulle, Außenminister Bidault und General Koenig die Kompromißbereitschaft ebenso unterschiedlich ausgeprägt war wie die politische und ökonomische Rationalität in der Bestimmung der nationalen Interessen. Zudem gab es nicht nur im Quai d'Orsay (Chauvel) erhebliche Widersprüche

25 Lattard, Gewerkschaften, S. 5 ff. Die Blockadepolitik war mehr Ausdruck der Hilflosigkeit als maliziöse Revanche für die Nichtbeteiligung an den Konferenzen von Yalta und Potsdam. 
gegen eine negative Blockadepolitik, die bloß verhinderte, aber nichts gestaltete. Vor allem die französische Kontrollratsgruppe unter General Koeltz war bereit, den USA in der entscheidenden Frage der Zentralverwaltungen sehr weit entgegenzukommen. Diese Widersprüchlichkeit, ja Doppeldeutigkeit produzierte erhebliche Irritationen, da alle in Berlin ausgehandelten Kompromisse in Paris (wenn nicht bereits in BadenBaden) verworfen wurden. Doch obwohl Frankreich in opportunistischer Illoyalität dabei notfalls auch sowjetische Schützenhilfe in Anspruch nahm, konnte es stets der weltpolitischen Rücksichtnahme und Solidarität seitens der Anglo-Amerikaner sicher sein. Die USA akzeptierten in Westdeutschland französische Alleingänge, Erpressungen, ungedeckte Vorteilnahmen und Brüskierungen, die auf sowjetischer Seite als eklatante Verletzung der Vier-Mächte-Vereinbarungen verurteilt wurden. Umgekehrt war die Sowjetunion zu keiner Zeit in der Lage, französische Offerten durch konkrete Zugeständnisse zu honorieren (z. B. in der Saarfrage), um den beargwöhnten westlichen Akkord zu durchbrechen. Das sollte sich 1948 sehr zum Nachteil für die Sowjets auswirken. Frankreich hätte prinzipiell den Kontrollrat jeder westlichen Trizonenlösung vorgezogen, obwohl es zu dieser Zeit durch eine isolierte Zonenlösung kaum noch nationalen Gewinn ziehen konnte, nachdem die Ertragfähigkeit der eigenen Besatzungszone infolge radikaler Ausbeutungspolitik längst erschöpft war. Es willigte schließlich in die Entscheidung zum Bruch ein, weil von der Sowjetunion kein entscheidendes Kooperationsangebot zu erwarten war, zumal nachdem mit dem Marshall-Plan die lukrativere Modernisierungsofferte vorlag, die sowohl Sicherheit vor Deutschland als auch Rekonstruktion notfalls obne Deutschland ermöglichte. Daran scheiterten in den folgenden Jahren alle sowjetischen Versuche, die offenkundig in erster Linie an die Adresse Frankreichs gerichtet waren, den Kontrollrat in der einen oder anderen Form wiederzubeleben.

Auch die britische Politik war erheblich widersprüchlicher, als es die jüngere Forschung gelegentlich behauptet hat. Gefangen zwischen einer wachsenden Abhängigkeit von den USA, machtpolitischer und ideologischer Distanz zur Sowjetunion und taktischer Annäherung an Frankreich war Deutschland das entscheidende Faustpfand gegenüber allen drei alliierten Konkurrenten. Sehr früh, schon im Herbst 1945, lief die interne Debatte über die „Revision von Potsdam“ auf eine Spaltung Deutschlands hinaus, die als die beste aller nachteiligen Lösungsvarianten galt. Doch es war keineswegs nur die taktische Überlegung, die Verantwortung für diesen Schritt nicht tragen zu wollen, die Bevin immer wieder zögern ließ. Die britische Regierung hat sich zweifellos als erste zum Bruch entschlossen, doch Bevins Weg zu diesem Entschluß war alles andere als geradlinig und konsequent. Clays erstes Bizonenangebot vom November 1945 lehnten die Briten noch ab, übernahmen dann zwar im Frühsommer 1946 die Initiative für ein derartiges Arrangement, doch mußte Bevin im Juli des gleichen Jahres von seinen Mitarbeitern sehr bedrängt werden, bis er schließlich grünes Licht gab: Offenkundig hatte er Bedenken, noch weiter in die Abhängigkeit von den USA zu geraten. Seine im Frühsommer 1946 verschiedentlich geäußerte Auffassung, man müsse der Sowjetunion die Schuld an der Teilung Deutschlands zuschieben, galt zunächst nur für den Fall, daß die politische Teilung tatsächlich unvermeidlich wurde. Er, wie die Franzosen auch, war an der auch wirtschaftlichen Teilung noch bis Ende 1947 nicht unbedingt interessiert. Doch nachdem Bevin einmal den Entschluß als unvermeidlich hingenommen hatte, ging er seinen Kurs mit ganzer Konsequenz, wie sein - 
im Sinne eines Kooperationsangebots an die USA - mehr taktisch-freiwilliges als erzwungenes Abrücken von der Sozialisierung der Ruhrindustrie unterstrich. Seine verschiedentlichen Initiativen (Pakt von Dünkirchen, Brüsseler Pakt, Marshall-Plan und NATO) dienten im folgenden dazu, wie sein Verhalten auf der entscheidenden Moskauer Außenministerkonferenz im März/April 1947 nahelegt, amerikanische Kompromißangebote an die Sowjetunion zu torpedieren, zugleich aber den USA die Sicherung Westeuropas vor Deutschland und der Sowjetunion aufzubürden.

Die Politik Clays ist ein repräsentatives Beispiel für die komplizierte Entschlußlage der Alliierten. Zweifellos hat Clay durch seine Ungeduld, seine herrische Persönlichkeit, aber auch durch seinen politischen Auftrag, die Besatzungsverwaltung frühzeitig an das State Department zu übergeben, wesentlich dazu beigetragen, arbeitsfähige Kompromißlösungen im Kontrollrat zu verhindern, indem er uni- oder bilaterale ErsatzArrangements suchte. Nachdem die amerikanischen Militärs unter Zugrundelegung optimaler Bedingungen (sowjetische Kooperation, Fortbestand deutscher zentraler Verwaltungsstellen und koordinierte europäische Rekonstruktion) ihre Vorstellung einer „Regierung nach Zonen“ und einer unverbindlichen, auf zonale Autonomie ausgerichteten Konstruktion des Kontrollrats hatten verwirklichen können, waren sie angesichts der katastrophalen Realitäten in Deutschland rasch davon überzeugt, daß zur Minimierung der Kosten wie ihrer politischen Verantwortung für die Zonenverwaltung gesamtdeutsche Lösungen erforderlich seien. Clays Politik 1945/46 entsprang der Doppeldeutigkeit seines Besatzungsauftrags, einerseits unilateral handlungsfähig bleiben zu müssen, andererseits seine Alliierten zur Kooperation zwingen zu wollen: u. a. durch den Reparationsstopp, der sich gegen die Sowjetunion und Frankreich gleichermaßen richtete und auch die westeuropäischen Gläubigernationen, inkl. Großbritannien, traf. Nicht zuletzt aus persönlicher Eitelkeit hat er sich zunächst dagegen gewehrt, daß die kontrollrats- bzw. deutschlandimmanenten Versuche, die Blockade zu lösen, durch „europäische“ Modelle abgelöst wurden. Aus dem gleichen Grund war er auf amerikanischer Seite einer der letzten, im Grunde durchgängig seit 1945, der bis zum Jahreswechsel 1947/48 für die Aufrechterhaltung des Kontrollrats plädierte, obwohl er selbst durch sein Ultimatum an die Sowjets längst einen Kurs eingeschlagen hatte, der zum Auszug der USA aus dem Kontrollrat am 10. bzw. 12. April 1948 hätte führen müssen. Am Beispiel der amerikanischen Politik ist eine totale Verkehrung der ursprünglichen Fronten zu erkennen, die - mit Abstufungen - für die anderen alliierten Nationen auch galt: Die Kräfte, die sich während der Planungsphase am entschiedensten gegen eine "zentralistische“ Konstruktion des Kontrollrats gewehrt hatten, d. h. vor allem die Militärs, waren nun (aus besatzungspragmatischen Gründen) zu Befürwortern der Einheit geworden, während die Diplomaten (aufgrund ideologischer Vorbehalte) ihre weitgefaßten gesamteuropäischen oder globalen Konzepte zugunsten der zonalen Autonomie und der Teilung Deutschlands aufgaben. Allerdings: Dem Scheitern des Kontrollrats, das aus deutscher Sicht der Beginn der Spaltung war, stand aus europäischer Sicht der Beginn einer neuen Ära gegenüber: Die Integration in supranationale Strukturen als „Ersatz“ hat sich als zukunftsträchtige und friedensfähige Alternative erwiesen. 